\title{
Dynamic Kinetic Resolution of Primary Amines with a Recyclable Pd Nanocatalyst for Racemization
}

\author{
Mahn-Joo Kim*, Won-Hee Kim, Kiwon Han, Yoon Kyung Choi, and Jaiwook Park* \\ Department of Chemistry, Pohang University of Science and Technology, San-31, Hyojadong, \\ Pohang 790-784, Korea.
}

\section{Supporting Information}

\section{Experimental Section}

\section{General Procedure for Racemization of $(S)-1$ a}

$(S)-1 \mathrm{a}(24 \mathrm{mg}, 0.20 \mathrm{mmol})$ was added to a suspension of Pd nanocatalyst (1.0 mol\%) in dry and degassed toluene $(0.10 \mathrm{M})$. The reaction mixture was stirred and heated to 70 ${ }^{\circ} \mathrm{C}$ under argon. After 12 or $24 \mathrm{~h}$, the reaction mixture was cooled to room temperature and filtered through a glass filter (pore size: 20-30 $\mu \mathrm{m}$ ). Filtrate was concentrated and analyzed by ${ }^{1} \mathrm{H}$ NMR spectroscopy, indicating that all of the substrate was consumed. The $\%$ ee was determined by HPLC (Whelk-O1, $n$-hexane/2-propanol $=80 / 20$, flow rate $=1.0 \mathrm{~mL} / \mathrm{min}, \mathrm{UV} 217 \mathrm{~nm}$ ) after converting to the corresponding amide (2a) by treatment with a few drops of acetic anhydride to the vial.

Table 1. Racemization of $(S)-1$ a.

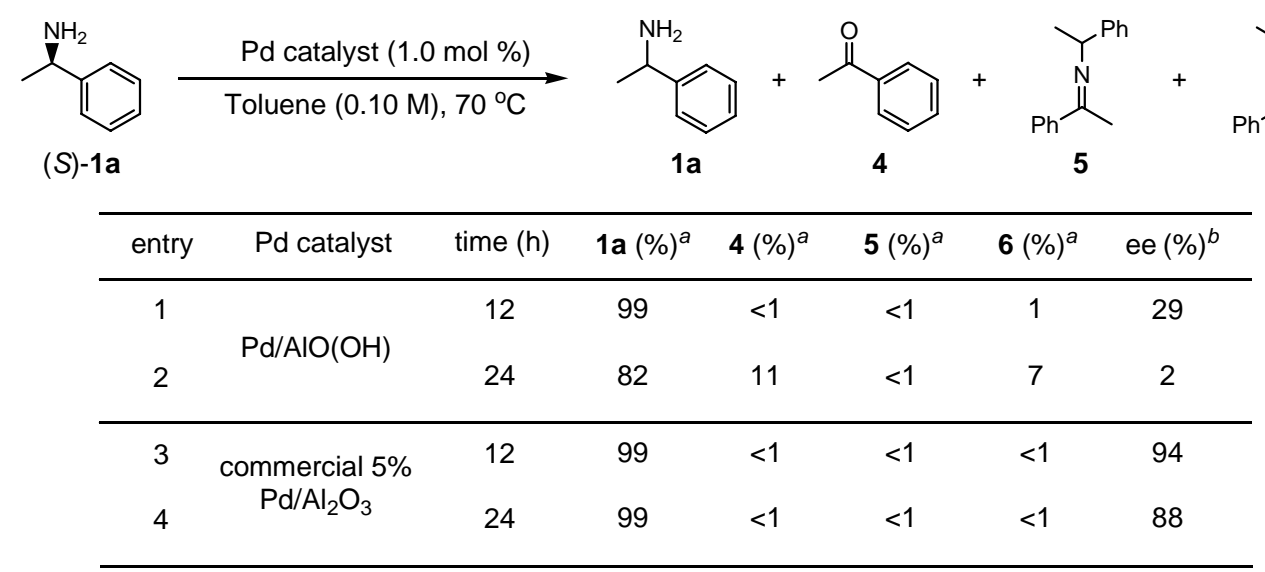

${ }^{a}$ Determined by ${ }^{1} \mathrm{H}$ NMR. ${ }^{b}$ Determined by HPLC (Whelk-O1). 


\section{General Procedure for Dynamic Kinetic Resolution of Benzyl Amines}

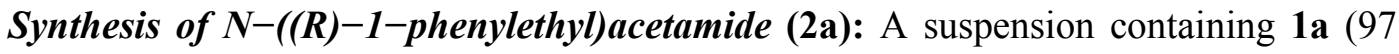
$\mathrm{mg}, 0.80 \mathrm{mmol})$, Pd nanocatalyst (1.0 mol\%), Novozym-435 (120 mg/mmol), ethyl acetate (3 equiv.), and molecular sieves $4 \AA$ (560 mg) in dry and degassed toluene $(0.10$ M) was stirred at $70{ }^{\circ} \mathrm{C}$ under argon atmosphere. After 3 days, the reaction mixture was cooled to room temperature and filtered through a glass filter (pore size: 20-30 $\mu \mathrm{m}$ ). Filtrate was concentrated and analyzed by ${ }^{1} \mathrm{H}$ NMR spectroscopy, indicating that all of the substrate was consumed. The mixture was subjected to a flash chromatography ( silica gel, $n$-hexane/ethyl acetate $=1 / 1)$ to provide $\mathbf{2 a}(119 \mathrm{mg}, 0.73 \mathrm{mmol}, 92 \%, 98 \%$ ee).

Synthesis of 2-methoxy-N-((R)-1-phenylethyl)acetamide (3a): A suspension containing 1a (39 mg, $0.30 \mathrm{mmol}$ ), Pd nanocatalyst (1.0 mol\%), Novozym-435 (15 $\mathrm{mg} / \mathrm{mmol})$, and ethyl methoxyacetate (1.7 equiv.) in dry and degassed toluene $(0.10 \mathrm{M})$ was stirred at $70{ }^{\circ} \mathrm{C}$ under argon atmosphere. After 3 days, the reaction mixture was cooled to room temperature and filtered through a glass filter (pore size: $20-30 \mu \mathrm{m}$ ). Filtrate was concentrated and analyzed by ${ }^{1} \mathrm{H}$ NMR spectroscopy, indicating that all of the substrate was consumed. The mixture was subjected to a flash chromatography ( silica gel, $n$-hexane/ethyl acetate $=1 / 1)$ to provide $3 \mathbf{a}(57 \mathrm{mg}, 0.29 \mathrm{mmol}, 98 \%, 99 \%$ ee).

\section{General Procedure for Dynamic Kinetic Resolution of Aliphatic Amines}

A suspension containing 1i (90 $\mathrm{mg}, 0.60 \mathrm{mmol})$, Pd nanocatalyst (12 $\mathrm{mol} \%$ ), Novozym-435 (120 mg/mmol) and ethyl acetate (3 equiv.) in dry and degassed toluene $(0.20 \mathrm{M})$ was stirred at $100{ }^{\circ} \mathrm{C}$ under $\mathrm{H}_{2}(1 \mathrm{~atm})$. After $4 \mathrm{~h}$, the reaction mixture was cooled to room temperature and filtered through a glass filter (pore size: $20-30 \mu \mathrm{m}$ ). Filtrate was concentrated and analyzed by GC spectroscopy, indicating that all of the substrate was consumed. The mixture was subjected to a flash chromatography (silica gel, $n$-hexane/ethyl acetate $=1 / 1)$ to provide $2 \mathbf{i}(109 \mathrm{mg}, 0.57 \mathrm{mmol}, 95 \%, 98 \%$ ee $)$.

\section{General Procedure for Recycling of Pd Nanocatalyst in Racemization of $(S)-1 i$}

$(S)-1 \mathbf{i}(30 \mathrm{mg}, 0.20 \mathrm{mmol})$ was added to a suspension of Pd nanaocatalyst ( $8.0 \mathrm{~mol} \%)$ in dry and degassed toluene $(0.20 \mathrm{M})$. The reaction mixture was stirred and heated to $100{ }^{\circ} \mathrm{C}$ under $\mathrm{H}_{2}(1 \mathrm{~atm})$. After $24 \mathrm{~h}$, the reaction mixture was cooled to room temperature and filtered through a glass filter (pore size: 20-30 $\mu \mathrm{m}$ ). Filtrate was concentrated and analyzed by ${ }^{1} \mathrm{H}$ NMR spectroscopy, indicating that all of the substrate 
was consumed. The $\%$ ee was determined by HPLC (Whelk-O1, $n$-hexane/2-propanol $=85 / 15$, flow rate $=0.50 \mathrm{~mL} / \mathrm{min}$, UV $217 \mathrm{~nm}$ ) after converting to the corresponding amide (2i) by treatment with a few drops of acetic anhydride to the vial. The separated catalysts were washed with ethyl acetate and dried in vacuo before reuse.

Table 2. Recycling of catalyst in racemization of $(S)-\mathbf{1 i}$.

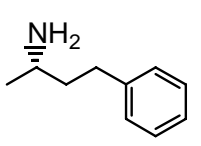

(S)-1i

(30 mg, $0.20 \mathrm{mmol})$ $>99 \%$ ee

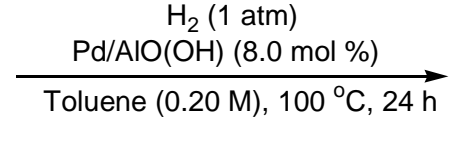

\begin{tabular}{cccc}
\hline Cycle & $\mathbf{1 i}(\%)^{a}$ & ${\text { byproducts }(\%)^{a}}^{a}$ & ee $(\%)^{b}$ \\
\hline 1 (fresh) & $>99$ & $<1$ & 3 \\
2 & $>99$ & $<1$ & 3 \\
3 & $>99$ & $<1$ & 3 \\
4 & $>99$ & $<1$ & 9 \\
5 & $>99$ & $<1$ & 4 \\
6 & $>99$ & $<1$ & 5 \\
7 & $>99$ & $<1$ & 5 \\
8 & $>99$ & $<1$ & 2 \\
9 & $>99$ & $<1$ & 9 \\
10 & $>99$ & $<1$ & 13
\end{tabular}

\footnotetext{
${ }^{a}$ Determined by GC. ${ }^{b}$ Determined by HPLC equipped with a chiral column
} (Whelk-O1) after converting to amide.

\section{General Procedure for Recycling of Catalysts in DKR}

A suspension containing 1i (30 mg, $0.20 \mathrm{mmol})$, Pd nanocatalyst (12 mol\%), Novozym-435 (120 mg/mmol) and ethyl acetate (3 equiv.) in dry and degassed toluene $(0.20 \mathrm{M})$ was stirred at $100{ }^{\circ} \mathrm{C}$ under $\mathrm{H}_{2}(1 \mathrm{~atm})$. After $4 \mathrm{~h}$, the reaction mixture was filtered through a glass filter $(20-30 \mu \mathrm{m})$ and the products in the filtrate were analyzed by GC and HPLC. The separated catalysts were washed with ethyl acetate and dried in vacuo before reuse. 
Table 3. Recycling of catalysts in DKR of $\mathbf{1 i}$.

\begin{tabular}{|c|c|c|c|c|}
\hline \multicolumn{4}{|c|}{$\begin{array}{l}\qquad \mathrm{H}_{2}(1 \mathrm{~atm}) \\
\text { Ethyl acetate }(53 \mathrm{mg}, 3.0 \text { equiv. }) \\
\text { vozym-435 }(24 \mathrm{mg}, 120 \mathrm{mg} / \mathrm{mmol}) \\
\text { Od/AlO }(\mathrm{OH})(297 \mathrm{mg}, 12 \mathrm{~mol} \%) \\
\stackrel{\text { Toluene }(0.20 \mathrm{M}), 100^{\circ} \mathrm{C}, 4 \mathrm{~h}}{\longrightarrow}\end{array}$} & \multirow{2}{*}{ ee $(\%)^{b}$} \\
\hline Cycle & Convn. $(\%)^{a}$ & $1 \mathbf{i}(\%)^{a}$ & $2 \mathbf{i}(\%)^{a}$ & \\
\hline 1 (fresh) & $>99$ & - & $>98$ & 99 \\
\hline 2 & $>99$ & - & $>98$ & 99 \\
\hline 3 & $>99$ & - & $>98$ & 98 \\
\hline 4 & $>99$ & - & $>98$ & 97 \\
\hline 5 & $>99$ & - & $>98$ & 96 \\
\hline 6 & $>99$ & - & $>98$ & 95 \\
\hline 7 & $>99$ & - & $>98$ & 94 \\
\hline 8 & 99 & 1 & $>98$ & 93 \\
\hline 9 & 93 & 7 & 93 & 93 \\
\hline 10 & 89 & 11 & 89 & 92 \\
\hline $11^{c}$ & $>99$ & - & $>98$ & 95 \\
\hline
\end{tabular}

\section{Characterization of Products}

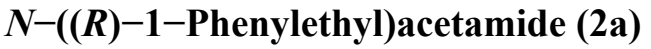

${ }^{1} \mathrm{H}$ NMR (300 MHz, $\left.\mathrm{CDCl}_{3}, \mathrm{ppm}\right): \delta 7.34-7.26(\mathrm{~m}, 5 \mathrm{H}), 5.85(\mathrm{~s}, 1 \mathrm{H}), 5.15-5.09(\mathrm{~m}$, $1 \mathrm{H}), 1.98$ (s, 3H), 1.49 (d, $J=6.88 \mathrm{~Hz}, 3 \mathrm{H}) ;{ }^{13} \mathrm{C} \mathrm{NMR}$ (75 MHz, $\left.\mathrm{CDCl}_{3}, \mathrm{ppm}\right): \delta 169.5$, $143.7,129.0,127.7,126.6,49.2,23.8,22.1$

HPLC condition

Whelk-O1, $n$-hexane $/ 2-$ propanol $=80 / 20$, flow rate $=1.0 \mathrm{~mL} / \mathrm{min}$, UV $217 \mathrm{~nm}$

$(S)-\mathbf{2 a}=8.19 \min ,(R)-\mathbf{2} \mathbf{a}=14.36 \min$

mp $97-100{ }^{\circ} \mathrm{C},[\alpha]^{25}+141\left(\mathrm{c}=1.0, \mathrm{CHCl}_{3}\right)$ 
(lit. mp $98-100{ }^{\circ} \mathrm{C},[\alpha]^{25}{ }_{\mathrm{D}}-140\left(\mathrm{c}=0.96, \mathrm{CHCl}_{3},(S)\right.$-isomer); Burk, M. J.; Wang, Y. M.; Lee, J. R. J. Am. Chem. Soc. 1996, 118, 5142.)

\section{2-Methoxy-N-((R)-1-phenylethyl)acetamide (3a)}

${ }^{1} \mathrm{H}$ NMR (300 MHz, $\left.\mathrm{CDCl}_{3}, \mathrm{ppm}\right): \delta 7.37-7.25(\mathrm{~m}, 5 \mathrm{H}), 6.76(\mathrm{~s}, 1 \mathrm{H}), 5.23-5.13(\mathrm{~m}$, $1 \mathrm{H}), 3.89$ (dd, $\left.J_{1}=19.65 \mathrm{~Hz}, J_{2}=14.97 \mathrm{~Hz}, 2 \mathrm{H}\right), 3.39$ (s, 3H), 1.51 (d, J=6.93 Hz, 3H);

${ }^{13} \mathrm{C} \mathrm{NMR}\left(75 \mathrm{MHz}, \mathrm{CDCl}_{3}, \mathrm{ppm}\right): \delta 168.6,143.0,128.7,127.4,126.1,72.0,59.1,48.0$, 21.9

HPLC condition

Whelk-O1, $n$-hexane $/ 2-$ propanol $=80 / 20$, flow rate $=2.0 \mathrm{~mL} / \mathrm{min}$, UV $217 \mathrm{~nm}$

$(S)-\mathbf{3 a}=4.97 \mathrm{~min},(R)-\mathbf{3 a}=11.86 \mathrm{~min}$

mp $59-60{ }^{\circ} \mathrm{C},[\alpha]^{25}+85.2\left(\mathrm{c}=0.5, \mathrm{CHCl}_{3}\right)$

Elemental analysis (VarioEL III CHN): calc. C 68.37, H 7.82, N 7.25; found C 68.31, H 7.82 N 7.24

\section{$N-((R)-1-p-$ Tolylethyl)acetamide (2b)}

${ }^{1} \mathrm{H}$ NMR (300 MHz, $\left.\mathrm{CDCl}_{3}, \mathrm{ppm}\right): \delta 7.19$ (d, $\left.J=8.08 \mathrm{~Hz}, 2 \mathrm{H}\right), 7.11(\mathrm{~d}, J=8.11 \mathrm{~Hz}$, 2H) $6.30(\mathrm{~s}, 1 \mathrm{H}), 5.10-4.98(\mathrm{~m}, 1 \mathrm{H}), 2.31(\mathrm{~s}, 3 \mathrm{H}), 1.93(\mathrm{~s}, 3 \mathrm{H}), 1.44(\mathrm{~d}, J=6.85 \mathrm{~Hz}$, $3 \mathrm{H}) ;{ }^{13} \mathrm{C}$ NMR $\left(75 \mathrm{MHz}, \mathrm{CDCl}_{3}, \mathrm{ppm}\right): \delta 169.9,141.0,137.5,129.9,126.7,49.1,23.9$, $22.4,21.6$

HPLC condition

Whelk-O1, $n$-hexane $/ 2-$ propanol $=80 / 20$, flow rate $=1.0 \mathrm{~mL} / \mathrm{min}, \mathrm{UV} 217 \mathrm{~nm}$

$(S)-\mathbf{2 b}=8.66 \mathrm{~min},(R)-\mathbf{2 b}=17.86 \mathrm{~min}$

mp $80-81{ }^{\circ} \mathrm{C},[\alpha]^{25}{ }_{\mathrm{D}}+146\left(\mathrm{c}=1.0, \mathrm{CHCl}_{3}\right)$

(lit. mp $80-82{ }^{\circ} \mathrm{C},[\alpha]^{25}{ }_{\mathrm{D}}+144\left(\mathrm{c}=1.03, \mathrm{CHCl}_{3}\right)$; Burk, M. J.; Wang, Y. M.; Lee, J. R. J. Am. Chem. Soc. 1996, 118, 5142.)

\section{2-Methoxy-N- ((R)-1-p-tolylethyl)acetamide (3b)}

${ }^{1} \mathrm{H}$ NMR (300 MHz, $\left.\mathrm{CDCl}_{3}, \mathrm{ppm}\right): \delta 7.22$ (d, $\left.J=8.13 \mathrm{~Hz}, 2 \mathrm{H}\right), 7.15$ (d, $\left.J=8.08 \mathrm{~Hz}, 2 \mathrm{H}\right)$, $6.73(\mathrm{~d}, J=5.47 \mathrm{~Hz}, 1 \mathrm{H}), 5.19-5.09(\mathrm{~m}, 1 \mathrm{H}), 3.88\left(\mathrm{dd}, J_{1}=20.46 \mathrm{~Hz}, J_{2}=14.95 \mathrm{~Hz}, 2 \mathrm{H}\right)$, 3.39 (s, 3H), 2.33 (s, 3H), 1.50 (d, J=6.91 Hz, 3H); $\left.{ }^{13} \mathrm{C} \mathrm{NMR} \mathrm{(75} \mathrm{MHz,} \mathrm{CDCl}_{3}, \mathrm{ppm}\right): \delta$ $168.5,140.0,137.1,129.3,126.1,72.0,59.1,47.8,21.8,21.0$

HPLC condition

Whelk-O1, $n$-hexane $/ 2$-propanol $=80 / 20$, flow rate $=2.0 \mathrm{~mL} / \mathrm{min}$. UV $217 \mathrm{~nm}$

$(S)-\mathbf{3 b}=5.04 \mathrm{~min},(R)-\mathbf{3 b}=14.28 \mathrm{~min}$

$\mathrm{mp} 44-46{ }^{\circ} \mathrm{C},[\alpha]^{25}+91.8\left(\mathrm{c}=0.5, \mathrm{CHCl}_{3}\right)$ 
Elemental analysis (VarioEL III CHN): calc. C 69.54, H 8.27, N 6.76; found C 69.39, H 8.34, N 6.64

N-((R)-1-(4-Methoxyphenyl)ethyl)acetamide (2c)

${ }^{1} \mathrm{H}$ NMR (300 MHz, $\left.\mathrm{CDCl}_{3}, \mathrm{ppm}\right): \delta 7.23$ (d, $\left.J=8.47 \mathrm{~Hz}, 2 \mathrm{H}\right), 6.85$ (d, $J=8.38 \mathrm{~Hz}$, 2H) $6.15(\mathrm{~s}, 1 \mathrm{H}), 5.10-4.95(\mathrm{~m}, 1 \mathrm{H}), 3.77(\mathrm{~s}, 3 \mathrm{H}), 1.93(\mathrm{~s}, 3 \mathrm{H}), 1.44(\mathrm{~d}, J=6.88 \mathrm{~Hz}$, $3 \mathrm{H}) ;{ }^{13} \mathrm{C}$ NMR $\left(75 \mathrm{MHz}, \mathrm{CDCl}_{3}, \mathrm{ppm}\right): \delta 169.7,159.3,136.0,128.0,114.5,55.9,48.7$, $23.9,22.3$

HPLC condition

Whelk-O1, $n$-hexane $/ 2-$ propanol $=80 / 20$, flow rate $=1.0 \mathrm{~mL} / \mathrm{min}$, UV $217 \mathrm{~nm}$

$(S)-\mathbf{2 c}=14.49 \mathrm{~min},(R)-\mathbf{2 c}=33.72 \mathrm{~min}$

mp $82-83{ }^{\circ} \mathrm{C},[\alpha]^{25}{ }_{\mathrm{D}}+145\left(\mathrm{c}=1.0, \mathrm{CHCl}_{3}\right)$

(lit. mp $82-84{ }^{\circ} \mathrm{C},[\alpha]^{25}{ }_{\mathrm{D}}+51.2\left(\mathrm{c}=0.11, \mathrm{CHCl}_{3}\right)$; Burk, M. J.; Wang, Y. M.; Lee, J. R. J. Am. Chem. Soc. 1996, 118, 5142.)

2-Methoxy-N-((R)-1-(4-methoxyphenyl)ethyl)acetamide (3c)

${ }^{1} \mathrm{H}$ NMR (300 MHz, $\left.\mathrm{CDCl}_{3}, \mathrm{ppm}\right): \delta 7.25$ (d, $\left.J=8.49 \mathrm{~Hz}, 2 \mathrm{H}\right), 6.87$ (d, $\left.J=8.76 \mathrm{~Hz}, 2 \mathrm{H}\right)$, $6.71(\mathrm{~d}, J=6.63 \mathrm{~Hz}, 1 \mathrm{H}), 5.18-5.09(\mathrm{~m}, 1 \mathrm{H}), 3.88\left(\mathrm{dd}, J_{1}=19.89 \mathrm{~Hz}, J_{2}=14.97 \mathrm{~Hz}, 2 \mathrm{H}\right)$, 3.79 (s, 3H), 3.39 (s, 3H), 1.50 (d, $J=6.93 \mathrm{~Hz}, 3 \mathrm{H}) ;{ }^{13} \mathrm{C} \mathrm{NMR}\left(75 \mathrm{MHz}, \mathrm{CDCl}_{3}, \mathrm{ppm}\right): \delta$ $168.5,158.9,135.1,127.4,114.0,72.0,59.1,55.3,47.5,21.7$

HPLC condition

Whelk-O1, $n$-hexane $/ 2-$ propanol $=80 / 20$, flow rate $=2.0 \mathrm{~mL} / \mathrm{min}$, UV $217 \mathrm{~nm}$

$(S)-\mathbf{3 c}=7.57 \mathrm{~min},(R)-\mathbf{3 c}=26.91 \mathrm{~min}$

mp $53-55^{\circ} \mathrm{C},[\alpha]^{25}+99.6\left(\mathrm{c}=0.5, \mathrm{CHCl}_{3}\right)$

Elemental analysis (VarioEL III CHN): calc. C 64.55, H 7.67, N 6.27; found C 64.47, H 7.74, N 6.22

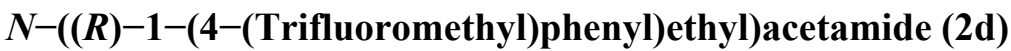

${ }^{1} \mathrm{H}$ NMR (300 MHz, $\left.\mathrm{CDCl}_{3}, \mathrm{ppm}\right): \delta 7.60$ (d, $\left.J=8.19 \mathrm{~Hz}, 2 \mathrm{H}\right), 7.42$ (d, $J=8.19 \mathrm{~Hz}$, 2H), $5.70(\mathrm{~s}, 1 \mathrm{H}), 5.21-5.11(\mathrm{~m}, 1 \mathrm{H}), 2.01(\mathrm{~s}, 3 \mathrm{H}), 1.50(\mathrm{~d}, J=6.96 \mathrm{~Hz}, 3 \mathrm{H}) ;{ }^{13} \mathrm{C}$ NMR (75 MHz, $\left.\mathrm{CDCl}_{3}, \mathrm{ppm}\right): \delta 169.2,147.3,126.4,125.6,125.5,48.5,29.7,23.3$, 21.8

HPLC condition

Whelk-O1, $n$-hexane $/ 2-$ propanol $=80 / 20$, flow rate $=1.0 \mathrm{~mL} / \mathrm{min}$, UV $217 \mathrm{~nm}$

$(S)-\mathbf{2 d}=8.59 \mathrm{~min},(R)-\mathbf{2 d}=16.47 \mathrm{~min}$

mp $103-105{ }^{\circ} \mathrm{C},[\alpha]^{25}+89.1\left(\mathrm{c}=1.0, \mathrm{CHCl}_{3}\right)$ 
(lit. mp $102-104{ }^{\circ} \mathrm{C},[\alpha]_{\mathrm{D}}^{25}-75.0\left(\mathrm{c}=0.12, \mathrm{CHCl}_{3},(S)\right.$-isomer); Burk, M. J.; Wang, Y. M.; Lee, J. R. J. Am. Chem. Soc. 1996, 118, 5142.)

\section{2-Methoxy-N-((R)-1-(4-(trifluoromethyl)phenyl)ethyl)acetamide (3d)}

${ }^{1} \mathrm{H}$ NMR (300 MHz, $\left.\mathrm{CDCl}_{3}, \mathrm{ppm}\right): \delta 7.59$ (d, $\left.J=8.20 \mathrm{~Hz}, 2 \mathrm{H}\right), 7.44$ (d, $\left.J=8.36 \mathrm{~Hz}, 2 \mathrm{H}\right)$, $6.82(\mathrm{~d}, J=6.59 \mathrm{~Hz}, 1 \mathrm{H}), 5.25-5.16(\mathrm{~m}, 1 \mathrm{H}), 3.90\left(\mathrm{dd}, J_{1}=18.27 \mathrm{~Hz}, J_{2}=15.03 \mathrm{~Hz}, 2 \mathrm{H}\right)$, 3.42 (s, 3H), 1.52 (d, $J=7.00 \mathrm{~Hz}, 3 \mathrm{H}) ;{ }^{13} \mathrm{C} \mathrm{NMR}\left(75 \mathrm{MHz}, \mathrm{CDCl}_{3}, \mathrm{ppm}\right): \delta 168.8,147.2$, $126.4,125.9,125.7,122.3,71.9,59.2,47.8,21.9$

HPLC condition

Whelk-O1, $n$-hexane $/ 2-$ propanol $=80 / 20$, flow rate $=2.0 \mathrm{~mL} / \mathrm{min}$, UV $217 \mathrm{~nm}$

$(S)-\mathbf{3 d}=6.95 \mathrm{~min},(R)-\mathbf{3 d}=23.26 \mathrm{~min}$ $\mathrm{mp} 82-84{ }^{\circ} \mathrm{C},[\alpha]^{25}+67.2\left(\mathrm{c}=0.5, \mathrm{CHCl}_{3}\right)$

Elemental analysis (VarioEL III CHN): calc. C 55.17, H 5.40, N 5.36; found C 55.36, H 5.46, N 5.44

\section{$N$-((R)-Phenylpropyl)acetamide (2e)}

${ }^{1} \mathrm{H}$ NMR (300 MHz, $\left.\mathrm{CDCl}_{3}, \mathrm{ppm}\right): \delta 7.35-7.25(\mathrm{~m}, 5 \mathrm{H}), 5.91(\mathrm{~s}, 1 \mathrm{H}), 4.91-4.83$ (m, $1 \mathrm{H}), 1.97(\mathrm{~s}, 3 \mathrm{H}), 1.88-1.78(\mathrm{~m}, 2 \mathrm{H}), 0.88(\mathrm{t}, J=7.39 \mathrm{~Hz}, 3 \mathrm{H}) ;{ }^{13} \mathrm{C}$ NMR $(75 \mathrm{MHz}$, $\left.\mathrm{CDCl}_{3}, \mathrm{ppm}\right): \delta 169.5,142.5,129.0,127.7,127.0,55.3,29.4,23.9,11.1$

HPLC condition

Chiralcel OD, $n$-hexane $/ 2-$ propanol $=93 / 7$, flow rate $=1.0 \mathrm{~mL} / \mathrm{min}, \mathrm{UV} 217 \mathrm{~nm}$

$(R)-\mathbf{2 e}=16.05 \mathrm{~min},(S)-\mathbf{2 e}=18.94 \mathrm{~min}$

mp $106-108{ }^{\circ} \mathrm{C},[\alpha]^{25}+123\left(\mathrm{c}=1.0, \mathrm{CHCl}_{3}\right)$

(lit. mp $105-107^{\circ} \mathrm{C},[\alpha]^{25}{ }_{\mathrm{D}}+135\left(\mathrm{c}=0.12, \mathrm{CHCl}_{3}\right)$; Burk, M. J.; Wang, Y. M.; Lee, J. R. J. Am. Chem. Soc. 1996, 118, 5142.)

\section{2-Methoxy $-N-((R)-$ phenylpropyl)acetamide (3e)}

${ }^{1} \mathrm{H}$ NMR (300 MHz, $\left.\mathrm{CDCl}_{3}, \mathrm{ppm}\right): \delta 7.37-7.23$ (m, 5H), $6.76(\mathrm{~d}, J=5.79 \mathrm{~Hz}, 1 \mathrm{H}), 4.97$ $-4.89(\mathrm{~m}, 1 \mathrm{H}), 3.89\left(\mathrm{dd}, J_{1}=24.90 \mathrm{~Hz}, J_{2}=15.00 \mathrm{~Hz}, 2 \mathrm{H}\right), 3.41(\mathrm{~s}, 3 \mathrm{H}), 1.90-1.80(\mathrm{~m}$, 2H), 0.90 (t, $J=7.38 \mathrm{~Hz}, 3 \mathrm{H}) ;{ }^{13} \mathrm{C}$ NMR $\left(75 \mathrm{MHz}, \mathrm{CDCl}_{3}, \mathrm{ppm}\right): \delta 168.8,141.9,128.6$, $127.4,126.6,72.0,59.1,54.1,29.3,10.7$

HPLC condition

Chiralcel OD, $n$-hexane $/ 2-$ propanol $=93 / 7$, flow rate $=1.0 \mathrm{~mL} / \mathrm{min}$, UV $217 \mathrm{~nm}$ $(R)-\mathbf{3 e}=8.22 \mathrm{~min},(S)-\mathbf{3 e}=14.13 \mathrm{~min}$ mp $71-72{ }^{\circ} \mathrm{C},[\alpha]^{25}+79.2\left(\mathrm{c}=0.5, \mathrm{CHCl}_{3}\right)$

Elemental analysis (VarioEL III CHN): calc. C 69.54, H 8.27, N 6.76; found C 69.23, H 
8.27, N 6.72

N-((R)-2,3-Dihydro-1H-inden-1-yl)acetamide (2f)

${ }^{1} \mathrm{H}$ NMR (300 MHz, $\left.\mathrm{CDCl}_{3}, \mathrm{ppm}\right): \delta 7.25-7.19(\mathrm{~m}, 4 \mathrm{H}), 6.29(\mathrm{~s}, 1 \mathrm{H}), 5.41-5.33(\mathrm{~m}$, $1 \mathrm{H}), 3.00-2.75(\mathrm{~m}, 2 \mathrm{H}), 2.53-2.47(\mathrm{~m}, 1 \mathrm{H}), 1.95(\mathrm{~s}, 3 \mathrm{H}), 1.80-1.73(\mathrm{~m}, 1 \mathrm{H}) ;{ }^{13} \mathrm{C}$ NMR (75 MHz, $\left.\mathrm{CDCl}_{3}, \mathrm{ppm}\right): \delta 170.1,143.7,143.5,128.4,127.2,125.2,124.4,55.2$, $34.5,30.6,23.9$

HPLC condition

Chiralcel OD,$n$-hexane $/ 2-$ propanol $=85 / 15$, flow rate $=1.0 \mathrm{~mL} / \mathrm{min}, \mathrm{UV} 217 \mathrm{~nm}$

$(S)-\mathbf{2 f}=11.70 \mathrm{~min},(R)-\mathbf{2 f}=13.18 \mathrm{~min}$

mp $151-153{ }^{\circ} \mathrm{C},[\alpha]^{25}+72.4\left(\mathrm{c}=1.0, \mathrm{CHCl}_{3}\right)$

(lit. $[\alpha]^{25}+74.4\left(\mathrm{c}=1.0, \mathrm{CHCl}_{3}\right)$; Zhang, Z.; Zhu, G.; Jiang, Q.; Xiao, D.; Zhang, X. J. Org. Chem. 1999, 64, 1774.)

2-Methoxy- $\mathbf{N}-((R)-2,3-$ dihydro-1H-inden-1-yl)acetamide (3f)

${ }^{1} \mathrm{H}$ NMR (300 MHz, $\left.\mathrm{CDCl}_{3}, \mathrm{ppm}\right): \delta 7.30-7.20(\mathrm{~m}, 4 \mathrm{H}), 6.74(\mathrm{~d}, J=5.61 \mathrm{~Hz}, 1 \mathrm{H}), 5.57$ $-5.50(\mathrm{~m}, 1 \mathrm{H}), 3.96(\mathrm{~s}, 2 \mathrm{H}), 3.39(\mathrm{~s}, 3 \mathrm{H}), 3.00-2.85(\mathrm{~m}, 2 \mathrm{H}), 2.64-2.59(\mathrm{~m}, 1 \mathrm{H})$, $1.87-1.80(\mathrm{~m}, 1 \mathrm{H}) ;{ }^{13} \mathrm{C} \mathrm{NMR}\left(75 \mathrm{MHz}, \mathrm{CDCl}_{3}, \mathrm{ppm}\right): \delta 169.3,143.4,142.9,128.0$, $126.8,124.8,124.0,72.0,59.1,53.9,34.0,30.3$

HPLC condition

Chiralcel OD, $n$-hexane $/ 2-$ propanol $=85 / 15$, flow rate $=1.0 \mathrm{~mL} / \mathrm{min}$, UV $217 \mathrm{~nm}$

$(S)-\mathbf{3 f}=7.68 \mathrm{~min},(R)-\mathbf{3 f}=10.98 \mathrm{~min}$ mp $50-52{ }^{\circ} \mathrm{C},[\alpha]^{25}+44.0\left(\mathrm{c}=0.5, \mathrm{CHCl}_{3}\right)$

Elemental analysis (VarioEL III CHN): calc. C 70.22, H 7.37, N 6.82; found C 69.52, H 7.39 , N 6.73

$N-((R)-1,2,3,4-$ Tetrahydronaphthalen-1-yl)acetamide (2g)

${ }^{1} \mathrm{H}$ NMR (300 MHz, $\left.\mathrm{CDCl}_{3}, \mathrm{ppm}\right): \delta 7.22-7.03(\mathrm{~m}, 4 \mathrm{H}), 6.29(\mathrm{~d}, J=7.85 \mathrm{~Hz}, 1 \mathrm{H})$, $5.12-5.07(\mathrm{~m}, 1 \mathrm{H}), 2.75-2.71(\mathrm{~m}, 2 \mathrm{H}), 2.01-1.93(\mathrm{~m}+\mathrm{s}, 4 \mathrm{H}), 1.84-1.73(\mathrm{~m}, 3 \mathrm{H})$;

${ }^{13} \mathrm{C}$ NMR (75 MHz, $\left.\mathrm{CDCl}_{3}, \mathrm{ppm}\right): \delta$ 169.6, 138.0, 137.1, 129.6, 129.1, 127.7, 126.6, $47.9,30.5,29.6,23.9,20.3$

HPLC condition

Chiralcel OD, $n$-hexane $/ 2-$ propanol $=90 / 10$, flow rate $=0.5 \mathrm{~mL} / \mathrm{min}, \mathrm{UV} 217 \mathrm{~nm}$

$(S)-\mathbf{2 g}=29.95 \mathrm{~min},(R)-\mathbf{2 g}=34.30 \mathrm{~min}$

mp $148-151{ }^{\circ} \mathrm{C},[\alpha]^{25}+83.6\left(\mathrm{c}=1.0, \mathrm{CHCl}_{3}\right)$ 
(lit. $[\alpha]^{25}{ }_{D}+106\left(\mathrm{c}=1.0, \mathrm{CHCl}_{3}\right)$; Zhang, Z.; Zhu, G.; Jiang, Q.; Xiao, D.; Zhang, X. J. Org. Chem. 1999, 64, 1774.)

\section{2-Methoxy-N-((R)-1,2,3,4-tetrahydronaphthalen-1-yl)acetamide (3g)}

${ }^{1} \mathrm{H}$ NMR (300 MHz, $\left.\mathrm{CDCl}_{3}, \mathrm{ppm}\right): \delta 7.28-7.09$ (m, 4H), 6.77 (d, $\left.J=7.35 \mathrm{~Hz}, 1 \mathrm{H}\right), 5.26$ $-5.21(\mathrm{~m}, 1 \mathrm{H}), 3.95(\mathrm{~s}, 2 \mathrm{H}), 3.38(\mathrm{~s}, 3 \mathrm{H}), 2.83-2.77(\mathrm{~m}, 2 \mathrm{H}), 2.10-2.03(\mathrm{~m}, 1 \mathrm{H})$, $1.89-1.79(\mathrm{~m}, 3 \mathrm{H}) ;{ }^{13} \mathrm{C} \mathrm{NMR}\left(75 \mathrm{MHz}, \mathrm{CDCl}_{3}, \mathrm{ppm}\right): \delta 168.8,137.6,136.5,129.2$, $128.6,127.3,126.3,72.0,59.1,46.8,30.2,29.2,20.0$

HPLC condition

Chiralcel OD, $n$-hexane $/ 2-$ propanol $=90 / 10$, flow rate $=0.5 \mathrm{~mL} / \mathrm{min}$, UV $217 \mathrm{~nm}$

$(S)-\mathbf{3 g}=15.83 \mathrm{~min},(R)-\mathbf{3 g}=20.20 \mathrm{~min}$ $\mathrm{mp} 86-87^{\circ} \mathrm{C},[\alpha]^{25}+65.8\left(\mathrm{c}=0.5, \mathrm{CHCl}_{3}\right)$

Elemental analysis (VarioEL III CHN): calc. C 71.21, H 7.81, N 6.39; found C 70.51, H 7.75, N 6.37

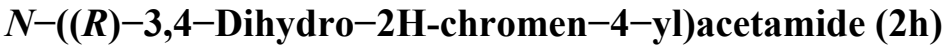

${ }^{1} \mathrm{H}$ NMR (300 MHz, $\left.\mathrm{CDCl}_{3}, \mathrm{ppm}\right): \delta 7.20-7.14(\mathrm{~m}, 2 \mathrm{H}), 6.89(\mathrm{t}, J=7.2 \mathrm{~Hz}, 1 \mathrm{H}), 6.82$ $(\mathrm{d}, J=8.13 \mathrm{~Hz}, 1 \mathrm{H}), 5.94(\mathrm{~s}, 1 \mathrm{H}), 5.14-5.07(\mathrm{~m}, 1 \mathrm{H}), 4.25-4.22(\mathrm{~m}, 1 \mathrm{H}), 4.17-4.10$ $(\mathrm{m}, 1 \mathrm{H}), 2.22-2.15(\mathrm{~m}, 1 \mathrm{H}), 2.07-2.00(\mathrm{~m}+\mathrm{s}, 4 \mathrm{H}) ;{ }^{13} \mathrm{C} \mathrm{NMR}\left(300 \mathrm{MHz}, \mathrm{CDCl}_{3}\right.$, ppm): $\delta 169.4,155.1,129.3,129.2,121.9,120.7,117.1,63.1,43.6,28.9,23.3$

HPLC condition

Chiralcel OD, $n$-hexane $/ 2-$ propanol $=85 / 15$, flow rate $=1.0 \mathrm{~mL} / \mathrm{min}$, UV $217 \mathrm{~nm}$

$(S)-\mathbf{2 h}=10.49 \mathrm{~min},(R)-\mathbf{2 h}=14.05 \mathrm{~min}$

mp $189-190{ }^{\circ} \mathrm{C},[\alpha]^{25}+79.2\left(\mathrm{c}=1.0, \mathrm{CHCl}_{3}\right)$

(lit. $[\alpha]^{25}{ }_{\mathrm{D}}+79.7$ (c=1.0, $\mathrm{CHCl}_{3}$ ); Zhang, Z.; Zhu, G.; Jiang, Q.; Xiao, D.; Zhang, X. J. Org. Chem. 1999, 64, 1774.)

\section{2-Methoxy-N-((R)-3,4-dihydro-2H-chromen-4-yl)acetamide (3h)}

${ }^{1} \mathrm{H}$ NMR (300 MHz, $\left.\mathrm{CDCl}_{3}, \mathrm{ppm}\right): \delta 7.21-7.16(\mathrm{~m}, 2 \mathrm{H}), 6.91(\mathrm{t}, J=7.26 \mathrm{~Hz}, 1 \mathrm{H}), 6.86$ $-6.82(\mathrm{~d}+\mathrm{s}, 2 \mathrm{H}), 5.24-5.16(\mathrm{~m}, 1 \mathrm{H}), 4.27-4.13(\mathrm{~m}, 2 \mathrm{H}), 3.94(\mathrm{~s}, 2 \mathrm{H}), 3.39(\mathrm{~s}, 3 \mathrm{H})$, $2.27-2.03$ (m, 2H); ${ }^{13} \mathrm{C}$ NMR (75 MHz, $\left.\mathrm{CDCl}_{3}, \mathrm{ppm}\right): \delta 169.0,155.1,129.3,129.2$, $121.8,120.9,117.2,71.9,63.2,59.1,43.0,29.1$

HPLC condition

Chiralcel OD, $n$-hexane $/ 2-$ propanol $=95 / 5$, flow rate $=0.5 \mathrm{~mL} / \mathrm{min}$, UV $217 \mathrm{~nm}$

$(S)-\mathbf{3 h}=14.34 \mathrm{~min},(R)-\mathbf{3 h}=20.70 \mathrm{~min}$ mp $137-138{ }^{\circ} \mathrm{C},[\alpha]^{25}+61.6\left(\mathrm{c}=0.5, \mathrm{CHCl}_{3}\right)$ 
Elemental analysis (VarioEL III CHN): calc. C 65.14, H 6.83, N 6.33; found C 65.06, H $6.83, \mathrm{~N} 6.31$

\section{N-((R)-4-Phenylbutan-2-yl)acetamide (2i)}

${ }^{1} \mathrm{H}$ NMR (300 MHz, $\left.\mathrm{CDCl}_{3}, \mathrm{ppm}\right): \delta 7.30-7.15$ (m, 5H), 5.47 (brs, $\left.1 \mathrm{H}\right), 4.10-4.00$ (m, 1H), $2.67-2.62(\mathrm{~m}, 2 \mathrm{H}), 1.93(\mathrm{~s}, 3 \mathrm{H}), 1.79-1.71(\mathrm{~m}, 2 \mathrm{H}), 1.17(\mathrm{~d}, J=6.6 \mathrm{~Hz}$, $3 \mathrm{H}) ;{ }^{13} \mathrm{C} \mathrm{NMR}\left(75 \mathrm{MHz}, \mathrm{CDCl}_{3}, \mathrm{ppm}\right): \delta 169.5,142.0,128.6,128.5,126.1,45.4,38.8$, 32.7, 23.7, 21.2

\section{HPLC condition}

Whelk-O1, $n$-hexane $/ 2-$ propanol $=85 / 15$, flow rate $=0.50 \mathrm{~mL} / \mathrm{min}$, UV $217 \mathrm{~nm}$

$(S)-\mathbf{2 i}=23.99 \mathrm{~min},(R)-\mathbf{2} \mathbf{i}=27.07 \mathrm{~min}$

$[\alpha]^{20}+41.3\left(\mathrm{c}=1.0, \mathrm{CH}_{2} \mathrm{Cl}_{2}\right)$

(lit. $[\alpha]_{\mathrm{D}}^{25}+45.5\left(\mathrm{c}=1.0, \mathrm{CH}_{2} \mathrm{Cl}_{2}\right)$; Paetzold, J.; Bäckvall, J. E. J. Am. Chem. Soc. 2005, 127, 17620.)

mp $71-72{ }^{\circ} \mathrm{C}$

(lit. mp $74-75{ }^{\circ} \mathrm{C}$; González-Sabín, J.; Gotor, V.; Rebolledo, F. Tetrahedron: Asymmetry 2002, 13, 1315.)

\section{N-((R)-1-Cyclohexylethyl)acetamide (2j)}

${ }^{1} \mathrm{H}$ NMR $\left(300 \mathrm{MHz}, \mathrm{CDCl}_{3}, \mathrm{ppm}\right): \delta 5.42$ (brs, $\left.1 \mathrm{H}\right), 3.88-3.81(\mathrm{~m}, 1 \mathrm{H}), 1.97(\mathrm{~s}, 3 \mathrm{H})$, $1.77-1.66(\mathrm{~m}, 5 \mathrm{H}), 1.36-1.13(\mathrm{~m}, 4 \mathrm{H}), 1.08(\mathrm{~d}, J=6.8 \mathrm{~Hz}, 3 \mathrm{H}), 1.01-0.93(\mathrm{~m}, 2 \mathrm{H})$; ${ }^{13} \mathrm{C} \mathrm{NMR}\left(75 \mathrm{MHz}, \mathrm{CDCl}_{3}\right.$, ppm): $\delta 169.4,49.6,43.3,29.3,26.6,26.4,23.8,18.1$

Chiral GC condition

BETA DEX 120, Supelco, injection temp. $220{ }^{\circ} \mathrm{C}$, detection temp. $300{ }^{\circ} \mathrm{C}$, oven initial temp. $120{ }^{\circ} \mathrm{C}(30 \mathrm{~min})$, rate $15{ }^{\circ} \mathrm{C} / \mathrm{min}$, oven final temp. $220{ }^{\circ} \mathrm{C}(10 \mathrm{~min})$

$(S)-\mathbf{2} \mathbf{j}=36.60 \mathrm{~min},(R)-\mathbf{2} \mathbf{j}=36.77 \mathrm{~min}$ mp $117-119{ }^{\circ} \mathrm{C},[\alpha]^{20}{ }_{\mathrm{D}}+19.1\left(\mathrm{c}=1.0, \mathrm{CH}_{2} \mathrm{Cl}_{2}\right)$

(lit. mp $101-102.5{ }^{\circ} \mathrm{C},[\alpha]^{25}+11.7(\mathrm{c}=5.27$, DMF); Mohan, A. T.; Conley, R. T. $J$. Org. Chem. 1969, 34, 3259.)

\section{$\mathrm{N}-((R)-$ Octan-2-yl)acetamide (2k)}

${ }^{1} \mathrm{H}$ NMR (300 MHz, $\left.\mathrm{CDCl}_{3}, \mathrm{ppm}\right): \delta 5.63$ (brs, $\left.1 \mathrm{H}\right) 4.00-3.90(\mathrm{~m}, 1 \mathrm{H}), 1.96(\mathrm{~s}, 3 \mathrm{H})$, $1.42-1.38(\mathrm{~m}, 2 \mathrm{H}), 1.27-1.17(\mathrm{~m}, 8 \mathrm{H}), 1.12(\mathrm{~d}, J=6.6 \mathrm{~Hz}, 3 \mathrm{H}), 0.88(\mathrm{t}, J=6.9 \mathrm{~Hz}$, $3 \mathrm{H}) ;{ }^{13} \mathrm{C} \mathrm{NMR}\left(75 \mathrm{MHz}, \mathrm{CDCl}_{3}, \mathrm{ppm}\right): \delta 169.5,45.5,37.1,31.9,29.3,26.1,23.6,22.7$, $21.1,14.2$

Chiral GC condition 
BETA DEX 120, Supelco, injection temp. $220{ }^{\circ} \mathrm{C}$, detection temp. $300{ }^{\circ} \mathrm{C}$, oven temp. $140{ }^{\circ} \mathrm{C}$

$(S)-\mathbf{2 k}=18.83 \mathrm{~min},(R)-\mathbf{2} \mathbf{k}=19.57 \mathrm{~min}$

$[\alpha]^{20}+6.6\left(\mathrm{c}=1.0, \mathrm{CH}_{2} \mathrm{Cl}_{2}\right)$

(lit. $[\alpha]_{\mathrm{D}}+4.4\left(\mathrm{c}=1.0, \mathrm{CH}_{2} \mathrm{Cl}_{2}\right)$; Paetzold, J.; Bäckvall, J. E. J. Am. Chem. Soc. 2005, 127, 17620.)

Not crystallized

\section{(S)-2-Acetamido-3-phenylpropanamide (2l)}

${ }^{1} \mathrm{H}$ NMR (300 MHz, DMSO, ppm): $\delta 8.04$ (s, 1H), $7.29-7.15$ (m, 5H), $4.45-4.37$ (m, $1 \mathrm{H}), 3.01-2.95(\mathrm{~m}, 1 \mathrm{H}), 2.76-2.68(\mathrm{~m}, 1 \mathrm{H}), 1.75(\mathrm{~s}, 3 \mathrm{H}),{ }^{13} \mathrm{C}$ NMR $(75 \mathrm{MHz}, \mathrm{DMSO}$, ppm): $\delta 173.2,169.0,138.2,129.0,127.9,126.1,53.7,37.6,22.5$

HPLC condition

Chiralcel OD, $n$-hexane $/ 2-$ propanol $=95 / 5$, flow rate $=1.0 \mathrm{~mL} / \mathrm{min}, \mathrm{UV} 217 \mathrm{~nm}$

$(S)-\mathbf{2 l}=55.04 \mathrm{~min},(R)-\mathbf{2} \mathbf{l}=59.15 \mathrm{~min}$

mp $177-179^{\circ} \mathrm{C},[\alpha]^{20}{ }_{\mathrm{D}}+36.6(\mathrm{c}=0.50, \mathrm{MeOH})$

(lit. mp $176-177{ }^{\circ} \mathrm{C},[\alpha]_{\mathrm{D}}^{25}+27(\mathrm{c}=0.10, \mathrm{MeOH})$; Huang, H. T.; Foster, R. J.; Niemann, C. J. Am. Chem. Soc. 1952, 74, 105.) 